\title{
An Interference Nulling Based Channel Independent Precoding for MIMO-OFDM Systems with Insufficient Cyclic Prefix
}

\author{
Yuansheng Jin and Xiang-Gen Xia, Fellow, IEEE
}

\begin{abstract}
In this paper, a new interference nulling based channel independent precoding for MIMO-OFDM systems of $n_{t}$ transmit and $n_{r}$ receive antennas with insufficient cyclic prefix $(\mathrm{CP})$ is proposed. By employing the notion of interference nulling, we show that our proposed channel independent precoding scheme can eliminate the inter-block interference (IBI) caused by the insufficient $\mathrm{CP}$ with higher bandwidth efficiency than the conventional zero-padded or a sufficient $\mathrm{CP}$ added block transmission system when $n_{r} \leq n_{t}$. It is also shown that when $n_{r}>n_{t}$, the IBI can be eliminated without the need of any zero-padding or adding CP or precoding when the OFDM block length is not too small.
\end{abstract}

Index Terms-MIMO-OFDM, precoding, interference alignment (IA), insufficient cyclic prefix (CP).

\section{INTRODUCTION}

I $\mathrm{N}$ the conventional MIMO-OFDM system, IDFT and CP insertion at the transmitter together with $\mathrm{CP}$ removal and DFT at the receiver help to convert an inter-symbol interference channel into several ISI free subchannels. The CP length is designed no less than the length of the channel impulse response (CIR) in order to eliminate the effects of the interblock interference (IBI) and inter-carrier interference (ICI). A considerably long CP is needed if the multipath delay spread is large, resulting in a substantial loss in both bandwidth and power efficiencies. In order to improve the transmission efficiency, MIMO-OFDM systems with insufficient CP have been studied significantly in the past, see, for example, [1][6], and OFDM/DMT systems with insufficient CP, in, for example, [7]-[9]. In [4], an ICI and ISI aware beamforming algorithm is proposed based on the optimal steering vector design that requires the channel state information at the transmitter (CSIT). In [7], a precoding is proposed to eliminate the distortion by processing the information symbols at the transmitter and it also requires the perfect CSIT. Instead of adding sufficient redundancies in the time domain, the

Paper approved by Cooperative and Heterogeneous Networks, the Editor for Cooperative and Heterogeneous Networks of the IEEE Communications Society. Manuscript received November 1, 2011; revised March 5 and June 4, 2012.

The authors are with the Department of Electrical and Computer Engineering, University of Delaware, Newark, DE 19716, USA. X.-G. Xia is also with Chonbuk National University, Jeonju, South Korea (e-mail: \{yjin, xxia\}@ee.udel.edu).

The authors' work was supported in part by the National Science Foundation (NSF) under Grant CCF-0964500, the World Class University (WCU) Program, the National Research Foundation, South Korea, and China International Cooperative Project No. 2009DFB10110.

Digital Object Identifier 10.1109/TCOMM.2012.092612.110740 technique proposed in [9] adds redundancies in the frequency domain by adding unused subcarriers. Some other techniques have been also proposed in [1]-[3], [5], [6], [8].

In a MIMO-OFDM system with insufficient $\mathrm{CP}$, if the IBI from the previous OFDM block can be separated and eliminated, it will be easier to detect the current OFDM block from the desired signal term and the ICI term both of which contain the information of the current OFDM symbol. Interference alignment (IA) [10]-[12] provides a novel concept to deal with interferences. The basic idea of IA is to use well-designed "beamforming" vectors at the transmitter such that the interference vectors are aligned at the receiver in one subspace which is disjoint from the signal subspace. As a result, the interference vectors are separated from the desired signal subspace and are limited in the minimum dimensions and therefore can be eliminated by the zero-forcing operator at the receiver. This basically provides an interference nulling technique.

In this paper, we adopt the notion of interference nulling in a MIMO-OFDM system and treat the IBI part as an interference channel. We propose a channel independent precoding scheme for a MIMO-OFDM system with insufficient $\mathrm{CP}$ or even no $\mathrm{CP}$. We show that our proposed precoding scheme can eliminate the IBI caused by the insufficient CP with a higher bandwidth efficiency than the conventional zero-padding or a sufficient $\mathrm{CP}$ adding when the number, $n_{r}$, of receive antennas is no more than the number, $n_{t}$, of transmit antennas, i.e., $n_{r} \leq n_{t}$. Interestingly, when $n_{t}=1$ and $n_{r}=1$, i.e., the single antenna case, in this paper, the IBI incurred from the insufficient $\mathrm{CP}$ can be aligned to a subspace of dimensions no more than a half of the difference of the ISI channel length and the insufficient $\mathrm{CP}$ length, thus the other half can be used for sending more information symbols. In this paper, it is also shown that when $n_{r}>n_{t}$, the IBI can be eliminated similarly without any zero-padding or adding $\mathrm{CP}$ or precoding when the OFDM block length is not too small.

The remainder of this paper is organized as follows. The SISO-OFDM and MIMO-OFDM system models are introduced in Section II. Our IA based precoding scheme is proposed in Section III. The relationships of the proposed IA based precoding with the existing block based transmissions are also discussed in Section III. Simulations results with some concrete examples are presented in Section IV to illustrate the theory developed in this paper. Conclusions are given in Section V. 
Some notations in this paper are defined as follows: Boldface upper-case letters denote matrices, boldface lower-case letters denote vectors. $\mathbf{0}_{m \times n}$ denotes a zero matrix with $m$ rows and $n$ columns. The operators $(\cdot)^{T}$ and $(\cdot)^{H}$ denote the transpose and Hermitian operations, respectively. $\mathbf{x}(i)$ and $[\mathbf{H}]_{i, j}$ denote the $i$ th entry of a vector $\mathbf{x}$ and the entry at the $i$ th row and the $j$ th column of a matrix $\mathbf{H}$, respectively. $\operatorname{rank}()$ stands for the column rank of a matrix and $\operatorname{span}\{\}$ stands for the linearly spanned space of the column vectors of a matrix. Lastly, for any $m \times n$ matrix $\mathbf{A}$, notation $\mathbf{A} \otimes \mathbf{I}_{k}$ denotes the $n k \times m k$ Kronecker product of matrix $\mathbf{A}$ and the identity matrix $\mathbf{I}_{k}$ of size $k$. $\operatorname{det}()$ stands for the determinant of a square matrix. $v$ is the CP length, $L$ is the CIR order, and $N$ is the number of subcarriers (or IDFT size).

\section{SySTEM MODEL}

To describe the signal model with concise but necessary notations, let us start with single antenna (single input and single output (SISO)) OFDM systems.

\section{A. SISO-OFDM Model}

Consider a SISO-OFDM system with $N$ subcarriers over a frequency-selective fading channel. The frequencyselective multipath channel is represented by a vector $\mathbf{h}=$ $[h(0), h(1), \cdots, h(L)]^{T}$, where $L+1$ is the length of the CIR and $L$ is called the order of the CIR. For convenience, these coefficients $h(l)$ are assumed i.i.d. complex Gaussian with 0 mean [23], [24]. In this paper, we assume that $N \geq L$. We use $\mathbf{r}_{k}=\left[r_{k}^{0}, r_{k}^{1}, \cdots, r_{k}^{N-1}\right]^{T}$ to denote the input signal vector of the $k$ th OFDM block. Let $\mathbf{W}_{N}$ denote the normalized IDFT matrix of size $N$ with entries $\left[\mathbf{W}_{N}\right]_{m, n}=(1 / \sqrt{N}) \exp (j 2 \pi m n / N)$. The IDFT operation is performed at the transmitter and changes the input signal from frequency domain to time domain. A CP of length $v$ is appended to each time domain vector. Since $\mathrm{CP}$ is generally insufficient in our study, we have $v \leq L$. The transmitted OFDM block is thus affected by both ICI and IBI components. After the insufficient $\mathrm{CP}$ is removed at the receiver, the time domain expression of the $k$ th received OFDM block is given, see, for example [7]:

$$
\mathbf{y}_{k}=(\mathbf{H}-\mathbf{A}) \mathbf{W}_{N} \mathbf{r}_{k}+\mathbf{B} \mathbf{W}_{N} \mathbf{r}_{k-1}+\mathbf{n}_{k},
$$

where $\mathbf{n}_{k}$ denotes the time domain received noise vector with the complex Gaussian distribution $\mathcal{C N}\left(\mathbf{0}, \sigma^{2} \mathbf{I}\right)$. The channel matrix $\mathbf{H}$ is a circulant matrix of size $N \times N$, the entry of which at the $m$ th row and the $n$th column is defined as $[\mathbf{H}]_{m, n}=h\left((m-n)_{N}\right)$, where $(l)_{N}$ means $l$ modulo $N$. A and $\mathbf{B}$ denote the $N \times N$ ICI and IBI components of the channel, respectively, defined as, see, for example [4], [9],

$$
\begin{gathered}
\mathbf{A}= \\
{\left[\begin{array}{ccc}
\mathbf{0}_{(L-v) \times(N-L)} & \mathbf{S} & \mathbf{0}_{(L-v) \times v} \\
\mathbf{0}_{(N-L+v) \times(N-L)} & \mathbf{0}_{(N-L+v) \times(L-v)} & \mathbf{0}_{(N-L+v) \times v}
\end{array}\right],} \\
\mathbf{B}=\left[\begin{array}{cc}
\mathbf{0}_{(L-v) \times(N-L+v)} & \mathbf{S} \\
\mathbf{0}_{(N-L+v) \times(N-L+v)} & \mathbf{0}_{(N-L+v) \times(L-v)}
\end{array}\right],
\end{gathered}
$$

where the $(L-v) \times(L-v)$ block matrix $\mathbf{S}$ is defined as:

$$
\mathbf{S}=\left[\begin{array}{cccc}
h(L) & h(L-1) & \cdots & h(v+1) \\
0 & h(L) & \cdots & h(v+2) \\
\vdots & \ddots & \ddots & \vdots \\
0 & \cdots & 0 & h(L)
\end{array}\right]
$$

In the above equations (2) and (3), matrices $\mathbf{A}$ and $\mathbf{B}$ are the time domain expressions derived under the assumption of perfect synchronization and a rectangular pulse shape. If $\mathrm{CP}$ length $v$ is larger than or equal to the CIR order $L, \mathbf{A}$ and $\mathbf{B}$ are both the all zero matrices. Therefore, no ICI or IBI exists in the received signal. For convenience, we denote $\mathbf{C}=\mathbf{H}-\mathbf{A}$ in (1), which is expressed explicitly in the $N \times N$ matrix in (5) at the bottom of the page. At the receiver, the time domain signal $\mathbf{y}_{k}$ in (1) is transformed into the frequency domain signal $\mathbf{z}_{k}$ by the DFT matrix $\mathbf{W}_{N}^{-1}$ of size $N$. We then have

$$
\mathbf{z}_{k}=\mathbf{W}_{N}^{-1} \mathbf{C} \mathbf{W}_{N} \mathbf{r}_{k}+\mathbf{W}_{N}^{-1} \mathbf{B} \mathbf{W}_{N} \mathbf{r}_{k-1}+\tilde{\mathbf{n}}_{k},
$$

where $\tilde{\mathbf{n}}_{k}=\mathbf{W}_{N}^{-1} \mathbf{n}_{k}$, and $\tilde{\mathbf{n}}_{k}$ is also distributed as $\mathcal{C N}\left(\mathbf{0}, \sigma^{2} \mathbf{I}\right)$.

Since we need to perform a precoding, signal $\mathbf{r}_{k}$ is the precoded output of an $N \times 1$ vector $\mathbf{x}_{k}$ of tentative information symbols (some of the components of $\mathbf{x}_{k}$ may be intentionally set to zero) passing through a precoding matrix $\mathbf{P}$ of size $N \times N$, i.e.,

$$
\mathbf{r}_{k}=\mathbf{P}_{\mathbf{x}_{k}}
$$

$$
\mathbf{C}=\left[\begin{array}{ccccccccc}
h(0) & 0 & \cdots & 0 & \cdots & 0 & h(v) & \cdots & h(1) \\
\vdots & \ddots & \ddots & & \ddots & \vdots & \vdots & \ddots & \vdots \\
\vdots & & \ddots & \ddots & & 0 & h(L) & & h(v) \\
\vdots & & & \ddots & \ddots & & 0 & \ddots & \vdots \\
\vdots & & & & \ddots & \ddots & & \ddots & h(L) \\
h(L) & & & & & \ddots & \ddots & & 0 \\
0 & \ddots & & & & & \ddots & \ddots & \vdots \\
\vdots & \ddots & \ddots & & & & & \ddots & 0 \\
0 & \cdots & 0 & h(L) & \cdots & \cdots & h(v-1) & \cdots & h(0)
\end{array}\right]
$$


We want to emphasize here that unlike the conventional precoding studies, the above precoding matrix $\mathbf{P}$ may not be full rank as we will see later. Since the IDFT matrix $\mathbf{W}_{N}$ is taken after the precoding matrix $\mathbf{P}$ and it is also a unitary matrix, the design of the precoding matrix $\mathbf{P}$ will be simplified if we consider $\mathbf{W}_{N} \mathbf{P}$ together. The time domain precoding matrix is defined as $\mathbf{Q} \triangleq \mathbf{W}_{N} \mathbf{P}$. After the design of $\mathbf{Q}$, the precoding matrix $\mathbf{P}$ can be obtained by multiplying with the inverse $\mathbf{W}_{N}^{-1}$. So, $\mathbf{P}$ and $\mathbf{Q}$ are equivalent and, in what follows, we call both $\mathbf{P}$ and $\mathbf{Q}$ precoders interchangeably.

From (6), the received frequency domain signal for the $k$ th OFDM block can be equivalently expressed as:

$$
\mathbf{z}_{k}=\mathbf{W}_{N}^{-1} \mathbf{C Q} \mathbf{x}_{k}+\mathbf{W}_{N}^{-1} \mathbf{B Q} \mathbf{x}_{k-1}+\tilde{\mathbf{n}}_{k}
$$

When insufficient $\mathrm{CP}$ is used, i.e., $v<L$, the IBI, $\mathbf{W}_{N}^{-1} \mathbf{B} \mathbf{Q} \mathbf{x}_{k-1}$, as shown in (8) causes that not all the information symbols in $\mathbf{x}_{k}$ can be solved freely. In Section III, we will first explore a SISO-OFDM example and then generalize our result to MIMO-OFDM to see how a precoder can be designed to help to solve for the variables in $\mathbf{x}_{k}$ and find how many such independent information symbols/variables, that corresponds to the rank of the precoder $\mathbf{Q}$, can be solved freely.

\section{B. MIMO-OFDM Model}

By considering a MIMO system with $n_{t}$ transmit, $n_{r}$ receive antennas, and by using the signal model in the SISO-OFDM system, the model of OFDM with insufficient CP is further extended to MIMO-OFDM in spatial multiplexing mode.

The overall input to the MIMO-OFDM system is noted by $\overline{\mathbf{r}}_{k}=\left[\left(\mathbf{r}_{k}^{0}\right)^{T},\left(\mathbf{r}_{k}^{1}\right)^{T}, \cdots,\left(\mathbf{r}_{k}^{N-1}\right)^{T}\right]^{T}$, where $\mathbf{r}_{k}^{i}$ denotes the $n_{t} \times 1$ vector for the $n_{t}$ transmit antennas at the $i$ th subcarrier, $0 \leq i \leq N-1$, in frequency domain. Next, the input vector $\overline{\mathbf{r}}_{k}$ is transformed into time domain signal by $n_{t}$ IDFT matrices of size $N$ at $n_{t}$ transmit antennas. The overall IDFT operation over $\overline{\mathbf{r}}_{k}$ can be represented by $\overline{\mathbf{W}} \triangleq \mathbf{W}_{N} \otimes \mathbf{I}_{n_{t}}$. At each transmit antenna, a CP of length $v$ is added to the input signal block and propagates via a multipath channel $\mathbf{h}_{i j}=\left[h_{i j}(0), h_{i j}(1), \cdots, h_{i j}(L)\right]^{T}$ between the $i$ th receive antenna and the $j$ th transmit antenna, where we assume that all the entries of $\mathbf{h}_{i j}$ are i.i.d. complex Gaussian random variables with 0 mean and the channel length, $L+1$, is identical for all the channels. We now define $n_{r} \times n_{t}$ channel matrices $\mathbf{H}(l)$, $l=0,1, \cdots, L$, as

$$
\mathbf{H}(l)=\left[\begin{array}{ccc}
h_{11}(l) & \cdots & h_{1 n_{t}}(l) \\
\vdots & \ddots & \vdots \\
h_{n_{r} 1}(l) & \cdots & h_{n_{r} n_{t}}(l)
\end{array}\right] .
$$

These matrices $\mathbf{H}(l), l=0,1, \cdots, L$, are the multipath channel matrices for the time domain vectors $\mathbf{r}_{k}^{i}$ serially transmitted at $n_{t}$ transmit antennas. Due to the randomness of the channel coefficients, all the matrices $\mathbf{H}(l)$ are of full rank almost surely.

At the receiver, the $\mathrm{CP}$ is removed and the overall time domain received block is given, for example [2]:

$$
\overline{\mathbf{y}}_{k}=\mathbf{C} \overline{\mathbf{W}} \overline{\mathbf{r}}_{k}+\mathbf{B} \overline{\mathbf{W}} \overline{\mathbf{r}}_{k-1}+\mathbf{n}_{k},
$$

where $\mathbf{n}_{k}$ is the $N n_{r} \times 1$ noise vector with the complex Gaussian distribution $\mathcal{C N}\left(\mathbf{0}, \sigma^{2} \mathbf{I}\right), \mathbf{C}$ and $\mathbf{B}$ of size $N n_{r} \times N n_{t}$ are the overall channel matrix and IBI matrix, respectively, constructed by stacking submatrices $\mathbf{H}(l)$ in (9) and shown in (11) at the bottom of the pape and (12).

$$
\mathbf{B}=\left[\begin{array}{cccccc}
\mathbf{0} & \cdots & \mathbf{0} & \mathbf{H}(L) & \cdots & \mathbf{H}(v+1) \\
\vdots & & & \ddots & \ddots & \vdots \\
\vdots & & & & \ddots & \mathbf{H}(L) \\
\vdots & & & & & \mathbf{0} \\
\vdots & & & & & \vdots \\
\mathbf{0} & \cdots & \cdots & \cdots & \cdots & \mathbf{0}
\end{array}\right]
$$

Before the signal detection, the DFT operation $\mathbf{W}_{N}^{-1} \otimes \mathbf{I}_{n_{r}}$ is applied to $\overline{\mathbf{y}}_{k}$ yielding the received signal in frequency domain. For this MIMO-OFDM system, the input vector $\overline{\mathbf{r}}_{k}$ is also the precoded output of information symbol vector $\overline{\mathbf{x}}_{k}=\left[\left(\mathbf{x}_{k}^{0}\right)^{T},\left(\mathbf{x}_{k}^{1}\right)^{T}, \cdots,\left(\mathbf{x}_{k}^{N-1}\right)^{T}\right]^{T}$ by an $N n_{t} \times N n_{t}$ precoding matrix $\mathbf{P}$, where $\mathbf{x}_{k}^{i}$ is the $n_{t} \times 1$ information symbol vector associated with $\mathbf{r}_{k}^{i}$ :

$$
\begin{aligned}
\overline{\mathbf{r}}_{k} & =\mathbf{P} \overline{\mathbf{x}}_{k} \\
& =\mathbf{P}\left[\left(\mathbf{x}_{k}^{0}\right)^{T},\left(\mathbf{x}_{k}^{1}\right)^{T}, \cdots,\left(\mathbf{x}_{k}^{N-1}\right)^{T}\right]^{T} .
\end{aligned}
$$

$$
\mathbf{C}=\left[\begin{array}{ccccccccc}
\mathbf{H}(0) & \mathbf{0} & \cdots & \mathbf{0} & \cdots & \mathbf{0} & \mathbf{H}(v) & \cdots & \mathbf{H}(1) \\
\vdots & \ddots & \ddots & & \ddots & \vdots & \vdots & \ddots & \vdots \\
\vdots & & \ddots & \ddots & & \mathbf{0} & \mathbf{H}(L) & & \mathbf{H}(v) \\
\vdots & & & \ddots & \ddots & & \mathbf{0} & \ddots & \vdots \\
\vdots & & & & \ddots & \ddots & & \ddots & \mathbf{H}(L) \\
\mathbf{H}(L) & & & & & \ddots & \ddots & & \mathbf{0} \\
0 & \ddots & & & & & \ddots & \ddots & \vdots \\
\vdots & \ddots & \ddots & & & & & \ddots & \mathbf{0} \\
0 & \cdots & \mathbf{0} & \mathbf{H}(L) & \cdots & \cdots & \mathbf{H}(v-1) & \cdots & \mathbf{H}(0)
\end{array}\right]
$$


Again, for the convenience of designing the precoding matrix, we consider the design of precoder $Q \triangleq \overline{\mathbf{W}} \mathbf{P}$. The precoding matrix $\mathbf{P}$ can then be obtained by multiplying $\mathbf{Q}$ with $\overline{\mathbf{W}}^{-1}=\mathbf{W}_{N}^{-1} \otimes \mathbf{I}_{n_{t}}$. So, both $\mathbf{P}$ and $\mathbf{Q}$ are called precoders interchangeably.

Then, we can represent the received frequency domain signal as

$$
\overline{\mathbf{z}}_{k}=\left(\mathbf{W}_{N}^{-1} \otimes \mathbf{I}_{n_{r}}\right) \mathbf{C Q} \overline{\mathbf{x}}_{k}+\left(\mathbf{W}_{N}^{-1} \otimes \mathbf{I}_{n_{r}}\right) \mathbf{B Q} \overline{\mathbf{x}}_{k-1}+\tilde{\mathbf{n}}_{k},
$$

in the $k$ th OFDM block.

\section{Channel IndePEndent PRECODING}

The main idea in the following is to design $\mathbf{Q}$ in (15) properly to align the received IBI in one subspace of as small dimension as possible which is also disjoint from the subspace occupied by the current information symbols. This coincides with the interference alignment concept [10]-[12], which means overlapping all the interference in one subspace and leaving the other subspace free from interference for the desired signal. We initialize the theory in this section by a simple SISO-OFDM example illustration and then provide our main results for MIMO-OFDM.

\section{A. SISO-OFDM Precoding Example}

Go back to the signal model (8) or equivalently,

$$
\mathbf{y}_{k}=\mathbf{C Q} \mathbf{x}_{k}+\mathbf{B Q} \mathbf{x}_{k-1}+\mathbf{n}_{k} .
$$

For the current $k$ th OFDM block, the signal to solve is $\mathbf{x}_{k}$ and $\mathbf{B Q} \mathbf{x}_{k-1}$ is the IBI. For convenience, assume that the additive noise $\mathbf{n}_{k}$ is negligible. In order to freely solve for $\mathbf{x}_{k}$ from (16), the space $\mathcal{V}_{\text {signal }}$ linearly spanned by the column vectors of $\mathbf{C Q}$ and the space $\mathcal{V}_{I B I}$ linearly spanned by the column vectors of $\mathrm{BQ}$ need to be disjoint.

For channel matrix $\mathbf{C}$ in (5), since its components $h(l)$ are i.i.d., the probability of its determinant, as a function of these random channel coefficients, to be zero is zero. This means that matrix $\mathbf{C}$ is full rank almost surely, i.e., its rank is $N$ almost surely. Its detailed proof is in Appendix I.

For the IBI matrix B in (3), due to its form in (3) and (4), its rank (or column rank) is $L-v$ almost surely similarly, where $v$ is the CP length.

Assume that the rank of the precoder $\mathbf{Q}$ is $N-d$ and it is aligned well enough such that the rank of the IBI matrix BQ is $L-v-d$ almost surely. For example, this can be achieved by setting the last $d$ row vectors of matrix $\mathbf{Q}$ all zero vectors. With such a precoder $\mathbf{Q}$, the rank of $\mathbf{C Q}$ is $N-d$ almost surely. In order for the spaces $\mathcal{V}_{\text {signal }}$ and $\mathcal{V}_{I B I}$ to be disjoint, the sum of their ranks has to be not more than the vector size $N$, i.e.,

$$
N-d+L-v-d \leq N
$$

With this dimension requirement, due to the randomness of the coefficients $h(l)$ in matrix $\mathbf{C}$ in (5) and matrix $\mathbf{B}$ in (3) and (4), the spaces $\mathcal{V}_{\text {signal }}$ and $\mathcal{V}_{I B I}$ are disjoint almost surely and $N-d$ variables in $\mathbf{C Q} \mathbf{x}_{k}$ (or in $\mathbf{x}_{k}$ ) can be solved freely.

What the inequality (17) means is that the precoding $\mathbf{Q}$ sacrifices $d$ dimensions and uses these $d$ dimensions to align the IBI into a space of dimension $L-v-d$. From (17), one can solve for $d$ :

$$
d \geq \frac{L-v}{2}
$$

and the smallest $d$ is

$$
d=\frac{L-v}{2},
$$

and in this case, the dimension of the space spanned by the IBI is also $(L-v) / 2$. Note that $v$ is the CP length and $L$ is CIR order. In the conventional OFDM system (or unprecoded OFDM system), additional $L-v$ zeros or redundant symbols are needed to make the IBI disappear. From the above analysis, only half of $L-v$ zeros or redundant symbols are needed to separate the spaces of the signal and the IBI for the signal to be solved freely.

To see a particular example for the above SISO-OFDM precoding idea, let us consider the case when $N=64$ subcarriers, CIR length $L+1=17$, i.e., $L=16$, and the insufficient $\mathrm{CP}$ length $v=12$. The time domain input to the OFDM system is precoded by a $64 \times 64$ precoding matrix $\mathbf{Q}$. From (18), $d=2$. Consider the following precoder

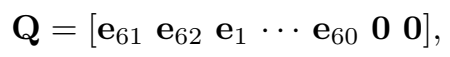

where $\mathbf{e}_{i} \triangleq[\overbrace{0, \cdots, 0}^{i-1}, 1,0, \cdots, 0]^{T}, 1 \leq i \leq 64$, is a set of $64 \times 1$ orthonormal vectors. It is easy to verify that $\operatorname{rank}(\mathbf{C Q})=62, \operatorname{rank}(\mathbf{B Q})=2$, and the column vectors of CQ are linearly independent of the only two nonzero column vectors $[h(0), 0, \cdots, 0]^{T}$ and $[h(0), h(1), 0, \cdots, 0]^{T}$ of $\mathbf{B Q}$, almost surely. In this example, 62 independent information symbols can be solved freely. With CP length $v=12$, in the conventional OFDM of block size $N=64,4$ more zeros or redundant symbols in the OFDM block are needed to completely eliminate the IBI, and thus only 60 independent information symbols can be included.

The detailed theory and the precoder construction will be given in the following subsection in a general form for MIMOOFDM systems.

\section{B. MIMO-OFDM Channel Independent Precoding}

In this subsection, we present a general theory and precoder construction for MIMO-OFDM systems by generalizing the idea discussed in the previous subsection.

For MIMO-OFDM systems, we can see from (10), (11) and (12) that the IBI term from the previous OFDM block needs to be suppressed and in the meantime the current OFDM block should be preserved. To do so, the same as the SISO-OFDM case, the basic idea is to design the precoding matrix $\mathbf{Q}$ such that the IBI can be aligned to an interference subspace which is disjoint from the signal subspace (spanned by signal vectors with the independent information symbols in $\overline{\mathbf{x}}_{k}$ ) that then can be solved freely as discussed in the preceding subsection. Furthermore, the dimensions of the interference subspace should be minimized, while the signal subspace occupies as many dimensions as possible in the receive signal space so that as many independent information symbols can be solved freely (without interference) as possible. For this purpose, the design criteria can be summarized as 
- $\operatorname{span}\{\mathbf{C Q}\} \bigcap \operatorname{span}\{\mathbf{B Q}\}=\{\mathbf{0}\}$

- $\operatorname{dim}(\mathbf{B Q})$ should be as small as possible;

- $\operatorname{dim}(\mathbf{C Q})$ should be as large as possible,

where $\operatorname{dim}$ means the dimension of the space linearly spanned by the column vectors of the matrix, which is the same as the column rank of the matrix. We next consider the problem in two different cases for the numbers $n_{t}$ and $n_{r}$ of transmit and receive antennas, respectively.

\section{Precoding When $n_{r} \leq n_{t}$}

In this case, from Appendix I matrix $\mathbf{C}$ is full row rank almost surely. The IBI matrix $\mathbf{B}$ in (12) is a block uppertriangular matrix and the upper right corner submatrix of $\mathbf{B}$ is $\mathbf{B}_{s}$ :

$$
\mathbf{B}_{s}=\left[\begin{array}{cccc}
\mathbf{H}(L) & \cdots & \cdots & \mathbf{H}(v+1) \\
0 & \ddots & & \vdots \\
\vdots & \ddots & \ddots & \vdots \\
0 & \cdots & 0 & \mathbf{H}(L)
\end{array}\right]
$$

which is of full (row) rank almost surely. Our goal is to design the precoding to cope with this $n_{r}(L-v) \times n_{t}(L-v)$ submatrix $\mathbf{B}_{s}$. To do so, the $n_{t} N \times n_{t} N$ precoding matrix $\mathbf{Q}$ is partitioned into submatrices:

$$
\mathbf{Q}=\left[\begin{array}{ll}
\mathbf{Q}_{1} & \mathbf{Q}_{2}
\end{array}\right]=\left[\begin{array}{ll}
\mathbf{Q}_{11} & \mathbf{Q}_{12} \\
\mathbf{Q}_{21} & \mathbf{Q}_{22}
\end{array}\right]
$$

where $\mathbf{Q}_{21}$ and $\mathbf{Q}_{22}$ are of the sizes $n_{t}(L-v) \times n_{1}$ and $n_{t}(L-v) \times n_{2}$, respectively. These two submatrices have the same number of rows as that of the columns of $\mathbf{B}_{s}$ and they are designed to suppress the IBI. The size of $\mathbf{Q}_{11}$ is $n_{t}(N-$ $L+v) \times n_{1}$ and the size of $\mathbf{Q}_{12}$ is $n_{t}(N-L+v) \times n_{2}$. Submatrices $\mathbf{Q}_{11}$ and $\mathbf{Q}_{12}$ should be designed to achieve more transmission rate, i.e., to have signal space dimension as large as possible. $\mathbf{Q}_{1}$ and $\mathbf{Q}_{2}$ are defined as $\mathbf{Q}_{1}=\left[\begin{array}{ll}\mathbf{Q}_{11}^{T} & \mathbf{Q}_{21}^{T}\end{array}\right]^{T}$, $\mathbf{Q}_{2}=\left[\begin{array}{ll}\mathbf{Q}_{12}^{T} & \mathbf{Q}_{22}^{T}\end{array}\right]^{T}$ and $n_{1}+n_{2}=n_{t} N$.

Lemma 1: If $\operatorname{span}\left\{\mathbf{Q}_{22}\right\} \subset \operatorname{span}\left\{\mathbf{Q}_{21}\right\}, n_{1} \leq n_{r}(L-v)$, and $\mathbf{Q}_{21}$ is full column rank, then, we have (almost surely)

$$
\operatorname{rank}(\mathbf{B Q})=\operatorname{rank}\left(\mathbf{B}_{s} \mathbf{Q}_{21}\right)=n_{1} .
$$

Proof:

$$
\mathbf{B Q}=\left[\begin{array}{cc}
\mathbf{0} & \mathbf{B}_{s} \\
\mathbf{0} & \mathbf{0}
\end{array}\right]\left[\begin{array}{ll}
\mathbf{Q}_{11} & \mathbf{Q}_{12} \\
\mathbf{Q}_{21} & \mathbf{Q}_{22}
\end{array}\right]=\left[\begin{array}{cc}
\mathbf{B}_{s} \mathbf{Q}_{21} & \mathbf{B}_{s} \mathbf{Q}_{22} \\
\mathbf{0} & \mathbf{0}
\end{array}\right] .
$$

Let $\mathbf{q}_{21}^{1}, \cdots, \mathbf{q}_{21}^{n_{1}}$ and $\mathbf{q}_{22}^{1}, \cdots, \mathbf{q}_{22}^{n_{2}}$ be the column vectors of $\mathbf{Q}_{21}$ and $\mathbf{Q}_{22}$, respectively. Then, $\mathbf{B}_{s} \mathbf{Q}_{21}$ and $\mathbf{B}_{s} \mathbf{Q}_{22}$ can be expressed as

$$
\begin{aligned}
\mathbf{B}_{s} \mathbf{Q}_{21} & =\left[\begin{array}{llll}
\mathbf{B}_{s} \mathbf{q}_{21}^{1} & \mathbf{B}_{s} \mathbf{q}_{21}^{2} & \cdots & \mathbf{B}_{s} \mathbf{q}_{21}^{n_{1}}
\end{array}\right], \\
\mathbf{B}_{s} \mathbf{Q}_{22} & =\left[\begin{array}{lllll}
\mathbf{B}_{s} \mathbf{q}_{22}^{1} & \mathbf{B}_{s} \mathbf{q}_{22}^{2} & \cdots & \mathbf{B}_{s} \mathbf{q}_{22}^{n_{2}}
\end{array}\right] .
\end{aligned}
$$

From $\operatorname{span}\left(\mathbf{Q}_{22}\right) \subset \operatorname{span}\left(\mathbf{Q}_{21}\right)$, we have

$$
\begin{gathered}
\operatorname{span}\left\{\mathbf{B}_{s} \mathbf{q}_{22}^{1}, \mathbf{B}_{s} \mathbf{q}_{22}^{2}, \cdots, \mathbf{B}_{s} \mathbf{q}_{22}^{n_{2}}\right\} \\
\subset \operatorname{span}\left\{\mathbf{B}_{s} \mathbf{q}_{21}^{1}, \mathbf{B}_{s} \mathbf{q}_{21}^{2}, \cdots, \mathbf{B}_{s} \mathbf{q}_{21}^{n_{1}}\right\} .
\end{gathered}
$$

Thus, we have proved the first equality in (22). The second equality in (22) is because matrix $\mathbf{B}_{s}$ in (20) has full row rank, $n_{r}(L-v)$, almost surely and $n_{1} \leq n_{r}(L-v)$.
In the MIMO-OFDM system with $n_{t}$ transmit and $n_{r}$ receive antennas and $N$ subcarriers, for each OFDM block there are total $n_{r} N$ linear equations after the CP removal. In order to be able to linearly solve for all the information symbols, the number of independent information symbols transmitted through the $n_{t}$ transmit antennas should be no more than $n_{r} N$. In what follows, we always impose this condition to the system. We next consider the following precodings.

(i) When $n_{t}(N-L+v)<n_{r} N$, we design the precoding matrix $\mathbf{Q}$ with the following properties:

1) $\operatorname{span}\left\{\mathbf{Q}_{22}\right\} \subset \operatorname{span}\left\{\mathbf{Q}_{21}\right\}$;

2) Take

$$
n_{1} \triangleq\left\lfloor\frac{n_{r} N-n_{t}(N-L+v)}{2}\right\rfloor .
$$

Both $\mathbf{Q}_{1}$ and $\mathbf{Q}_{21}$ are full column rank;

3) Submatrix $\mathbf{Q}_{12}$ has $n_{t}(N-L+v)$ linearly independent column vectors, say the first $n_{t}(N-L+v)$ column vectors in $\mathbf{Q}_{12}$ are linearly independent, and matrix $\mathbf{Q}_{2}$ has column rank $n_{t}(N-L+v)$;

4) Any non-all-zero linear combinations of the first $n_{t}(N-$ $L+v)$ column vectors of $\mathbf{Q}_{2}$ do not belong to $\operatorname{span}\left\{\mathbf{Q}_{1}\right\}$.

To show the feasibility of the above precoder $\mathbf{Q}$ design, we need to show the feasibility of the design of the submatrices $\mathbf{Q}_{21}$ and $\mathbf{Q}_{1}$ that are full column rank required in 2) and the feasibility of the design of the submatrix $\mathbf{Q}_{12}$ that can have $n_{t}(N-L+v)$ linearly independent columns required in 3$)$. From (23) and $n_{r} \leq n_{t}$, we have the following inequality:

$n_{1}=\left\lfloor\frac{n_{r} N-n_{t}(N-L+v)}{2}\right\rfloor \leq\left\lfloor\frac{n_{r}(L-v)}{2}\right\rfloor \leq n_{t}(L-v)$.

This means that the number of columns of $\mathbf{Q}_{21}$ (also $\mathbf{Q}_{1}$ ) is no more than the number of rows of $\mathbf{Q}_{21}$ (also $\mathbf{Q}_{1}$ ), which implies that a full column rank $\mathbf{Q}_{21}$ (also $\mathbf{Q}_{1}$ ) can be designed. In the meantime, we have

$$
n_{2}=n_{t} N-n_{1} \geq n_{t} N-n_{t}(L-v)=n_{t}(N-L+v) .
$$

This shows that $\mathbf{Q}_{12}$ is a fat matrix. Since its number of columns, $n_{2}$, is greater than or equal to its number of rows, $n_{t}(N-L+v), \mathbf{Q}_{12}$ can always be designed to be full row rank, which means that the column rank, i.e., the number of linearly independent columns, can be $n_{t}(N-L+v)$.

(ii) When $n_{t}(N-L+v) \geq n_{r} N$, the precoding matrix $\mathbf{Q}$ is designed as follows. We design $\left[\begin{array}{ll}\mathbf{Q}_{11} & \mathbf{Q}_{12}\end{array}\right]$ to be full row rank that is $n_{t}(N-L+v)$, and set $\mathbf{Q}_{21}=\mathbf{Q}_{22}=\mathbf{0}$. Thus,

$$
\mathbf{Q}=\left[\begin{array}{cc}
\mathbf{Q}_{11} & \mathbf{Q}_{12} \\
\mathbf{0} & \mathbf{0}
\end{array}\right] \text {. }
$$

With the above precoding, we have the following result.

Theorem 1: For the insufficient CP MIMO-OFDM system with $n_{r} \leq n_{t}$, the total number of independent information symbols can be solved by the zero-forcing operator during each OFDM block is

$$
\left\{\begin{array}{c}
n_{t}(N-L+v)+\left\lfloor\frac{n_{r} N-n_{t}(N-L+v)}{2}\right\rfloor, \\
\quad \text { if } n_{t}(N-L+v)^{2}<n_{r} N, \\
n_{r} N, \quad \text { if } n_{t}(N-L+v) \geq n_{r} N .
\end{array}\right.
$$

Proof: We first consider the case when $n_{t}(N-L+v)<$ $n_{r} N$. 
From the above precoder property 2) we have almost surely (since matrix $\mathbf{C}$ is almost surely full row rank and from (23), one can see that the number of rows of $\mathbf{C}, n_{r} N>n_{1}$ )

$$
\operatorname{rank}\left(\mathbf{C Q}_{1}\right)=n_{1}
$$

From the precoder property 3 ) and the number of rows of $\mathbf{C}$, $n_{r} N$ is greater than $n_{t}(N-L+v)$, we can derive (almost surely)

$$
\operatorname{rank}\left(\mathbf{C Q}_{2}\right)=n_{t}(N-L+v) .
$$

From the precoder properties 3) and 4), we know that $\operatorname{span}\left\{\mathbf{Q}_{1}\right\} \bigcap \operatorname{span}\left\{\mathbf{Q}_{2}\right\}=\{\mathbf{0}\}$. As a result,

$$
\begin{aligned}
\operatorname{rank}(\mathbf{C Q}) & =\operatorname{rank}\left(\mathbf{C Q} \mathbf{Q}_{1}\right)+\operatorname{rank}\left(\mathbf{C Q}_{2}\right) \\
& =n_{1}+n_{t}(N-L+v) .
\end{aligned}
$$

With the properties 1) and 2) and (24), we have the result (22) in Lemma 1, i.e.,

$$
\operatorname{rank}(\mathbf{B Q})=n_{1}
$$

Thus,

$$
\begin{array}{r}
\operatorname{rank}(\mathbf{B Q})+\operatorname{rank}(\mathbf{C Q})=2 n_{1}+n_{t}(N-L+v) \\
=2\left\lfloor\frac{n_{r} N-n_{t}(N-L+v)}{2}\right\rfloor+n_{t}(N-L+v) \leq n_{r} N .
\end{array}
$$

From Appendix II, the following equation holds almost surely:

$$
\operatorname{span}\{\mathbf{C Q}\} \bigcap \operatorname{span}\{\mathbf{B Q}\}=\{\mathbf{0}\} \text {. }
$$

With the zero-forcing operator, the number of independent information symbols that can be solved equals to the dimension of the desired signal subspace that is the column rank of $\mathrm{CQ}$, i.e.,

$$
n_{t}(N-L+v)+\left\lfloor\frac{n_{r} N-n_{t}(N-L+v)}{2}\right\rfloor,
$$

in one OFDM block for all $n_{t}$ transmit antennas.

We next consider the case when $n_{t}(N-L+v) \geq$ $n_{r} N$. In this case, from the precoder design, we have $\operatorname{rank}\left(\left[\begin{array}{ll}\mathbf{Q}_{11} & \mathbf{Q}_{12}\end{array}\right]\right) \geq n_{t}(N-L+v) \geq n_{r} N$. Thus, $\operatorname{rank}(\mathbf{C Q})=n_{r} N$, since $\mathbf{C}$ is full row rank almost surely. Furthermore, $\mathbf{B Q}=\mathbf{0}$, i.e., the IBI is totally eliminated. Since there are total $n_{r} N$ linear equations, the number of independent information symbols transmitted through the $n_{t}$ transmit antennas should be no more than $n_{r} N$ to linearly solve for all the information symbols.

From (30) in the above proof, one can see that the number of a part of independent information symbols, $n_{1}$, can not be larger, since otherwise the sum of the numbers of columns in matrices $\mathbf{B Q}$ and $\mathbf{C Q}$ will be more than the number of rows, i.e., will exceed the receive signal space dimension. This implies that $n_{1}=\left\lfloor\frac{n_{r} N-n_{t}(N-L+v)}{2}\right\rfloor$ in (23) in 2) is optimal already. We next present a precoder example for $\mathbf{Q}$.

Example: For the $n_{t} N$ dimensional vector space, $\left\{\mathbf{e}_{1}, \mathbf{e}_{2}, \cdots, \mathbf{e}_{n_{t} N}\right\}$ is a set of $n_{t} N \times 1$ orthonormal basis elements where $\mathbf{e}_{i}=[\underbrace{0, \cdots, 0}_{i-1}, 1,0, \cdots, 0]^{T}$ is as before. Let

$$
\begin{aligned}
& \mathbf{Q}_{2}=\left[\mathbf{e}_{1}, \cdots, \mathbf{e}_{n_{t}(N-L+v)}, \mathbf{e}_{1}, \cdots, \mathbf{e}_{1}\right] \\
& =[\mathbf{e}_{1}, \cdots, \mathbf{e}_{n_{\alpha}}, \underbrace{\mathbf{e}_{1}, \cdots, \mathbf{e}_{1}}_{n_{t} N-n_{1}-n_{\alpha}}], \\
& \mathbf{Q}_{1}=\left[\mathbf{e}_{n_{t}(N-L+v)+1}, \cdots, \mathbf{e}_{n_{t}(N-L+v)+n_{1}}\right] \\
& =\left[\mathbf{e}_{n_{\alpha}+1}, \cdots, \mathbf{e}_{n_{\beta}}\right] \text {, } \\
& n_{\alpha} \triangleq n_{t}(N-L+v), n_{\beta} \triangleq n_{\alpha}+n_{1} \text {. }
\end{aligned}
$$

In this example, $\mathbf{Q}_{12}$ contains an identity matrix of size $n_{\alpha}$, i.e.,

$$
\mathbf{Q}_{12}=\left[\mathbf{I}_{n_{\alpha}}, \tilde{\mathbf{e}}_{1}, \cdots, \tilde{\mathbf{e}}_{1}\right]
$$

where $\tilde{\mathbf{e}}_{1}=[1,0, \cdots, 0]^{T}$ is an $n_{\alpha} \times 1$ vector. Thus, the precoder property 3 ) is satisfied. The orthogonality among $\mathbf{e}_{i}, i=1, \cdots, n_{t} N$, ensures the precoder properties 2) and 4). Lastly, $\mathbf{Q}_{22}$ is a zero matrix now and therefore $\operatorname{span}\left\{\mathbf{Q}_{22}\right\} \subset$ $\operatorname{span}\left\{\mathbf{Q}_{21}\right\}$, i.e., the precoder property 1) holds as well. Interestingly, the submatrix $\mathbf{Q}_{21}$ here is not a zero matrix. Also note that, the last $\left(n_{t} N-n_{1}-n_{\alpha}\right)$ columns in $\mathbf{Q}_{2}$ in (32) do not have to be all $\mathbf{e}_{1}$ and in fact, they can be any linear combinations of the first $n_{\alpha}$ vectors $\mathbf{e}_{1}, \cdots, \mathbf{e}_{n_{\alpha}}$ in $\mathbf{Q}_{2}$ as mentioned earlier, for example, all zero vectors, which does not affect the precoder design properties 1)-4). It is noticeable that the SISO-OFDM precoder example in (19) satisfies these four properties and exhibits a special case of the above precoder design.

Using the precoder defined in (32)-(33), we are able to specify the final signals to be transmitted at the $n_{t}$ transmit antennas when $x_{k}(n)$ are independent information symbols as follows. In this example, $n_{\beta}$ is the number of total independent information symbols to be transmitted through $n_{t}$ transmit antennas in every data block of length $N$ where there are total $n_{t} N-n_{\beta}$ zeros inserted in the block. Every data block of length $N$ is preceded by a $\mathrm{CP}$ of length $v$. The overall transmission block length is $N+v$.

Firstly, for each time index $k$, all $n_{\beta}$ independent information symbols, $x_{k}(n), 0 \leq n \leq n_{\beta}-1$, are padded with $n_{t} N-n_{\beta}$ zeros to yield

$$
\overline{\mathbf{x}}_{k}=\left[x_{k}(0), x_{k}(1), \cdots, x_{k}\left(n_{\beta}-1\right), 0, \cdots, 0\right]^{T},
$$

which is then fed to $\mathbf{Q} \overline{\mathbf{x}}_{k}$. This is that each of the $n_{t}$ transmit antennas alternatively takes one symbol from $\overline{\mathbf{x}}_{k}$ consecutively for $N$ times to fill its data block of length $N$. To see this, for convenience, let us define

$$
\theta \triangleq\left\lfloor\frac{n_{\beta}}{n_{t}}\right\rfloor \text { and } n_{t 1} \triangleq n_{\beta}-\theta n_{t} .
$$

In this manner, for the first $n_{t 1}$ transmit antennas, the $j$ th antenna, $j=0, \cdots, n_{t 1}-1$, transmits the following data block of length $N$ :

$$
\begin{gathered}
\overline{\mathbf{x}}_{k}^{j}= \\
{\left[x_{k}\left(n_{t} \cdot 0+j\right), x_{k}\left(n_{t} \cdot 1+j\right), \cdots, x_{k}\left(n_{t} \cdot \theta+j\right), 0, \cdots, 0\right]^{T},}
\end{gathered}
$$

where $N-(\theta+1)$ zeros are inserted. For the remaining $n_{t}-$ $n_{t 1}$ transmit antennas, the $j$ th antenna, $j=n_{t 1}, \cdots, n_{t}-1$, transmits the following data block of length $N$ : 


$$
\begin{gathered}
\overline{\mathbf{x}}_{k}^{j}= \\
{\left[x_{k}\left(n_{t} \cdot 0+j\right), x_{k}\left(n_{t} \cdot 1+j\right) \cdots x_{k}\left(n_{t}(\theta-1)+j\right), \cdots, 0\right]^{T},}
\end{gathered}
$$

where $N-\theta$ zeros are inserted. Then, the last $v$ symbols of $\overline{\mathbf{x}}_{k}^{j}$ for each antenna $j$ are added in the front of the data block as a CP (usually insufficient in our IA based precoding) for every $k$ th data block of length $N$. Finally, the $k$ th transmission block of total length $N+v$ with $\mathrm{CP}$ is transmitted through all the $n_{t}$ antennas. More detailed examples are given in the simulations in the next section.

For a general precoder $\mathrm{Q}$, according to Theorem 1, the number of independent information symbols that can be put in $\overline{\mathbf{x}}_{k}$ in (13) and can be solved with the IBI free is shown in (26), which can be encoded as follows. To illustrate it, let us first see the previous example where the precoder $\mathbf{Q}=\left[\begin{array}{ll}\mathbf{Q}_{1} & \mathbf{Q}_{2}\end{array}\right]$ is shown in (32)-(33). In this example, all the $n_{1}$ columns in $\mathbf{Q}_{1}$ plus the first $n_{\alpha}$ columns, $\mathbf{e}_{1}, \cdots, \mathbf{e}_{n_{\alpha}}$, in $\mathbf{Q}_{2}$ are linearly independent. We can consider them altogether denoted as $\mathbf{Q}_{u}=\left[\mathbf{e}_{n_{\alpha}+1}, \cdots, \mathbf{e}_{n_{\beta}}, \mathbf{e}_{1}, \cdots, \mathbf{e}_{n_{\alpha}}\right]$. This submatrix $\mathbf{Q}_{u}$ corresponds to the first $n_{\beta}$ independent information symbols in $\overline{\mathbf{x}}_{k}$. The last $n_{t} N-n_{\beta}$ columns of $\mathbf{Q}_{2}$ are denoted as $\mathbf{Q}_{z}=\left[\mathbf{e}_{1}, \cdots, \mathbf{e}_{1}\right]$. The last $n_{t} N-n_{\beta}$ signals in $\overline{\mathbf{x}}_{k}$ in (13) corresponding to this submatrix $\mathbf{Q}_{z}$ are aligned in the space spanned by the IBI and can not be solved freely and thus these signals are set to zeros in $\overline{\mathbf{x}}_{k}$. As a result, the precoder $\mathbf{Q}$ is structured as

$$
\mathbf{Q}=\left[\begin{array}{ll}
\mathbf{Q}_{u} & \mathbf{Q}_{z}
\end{array}\right]
$$

which can be obtained similarly if the design of $\mathbf{Q}$ follows the general design properties 1) - 4) obtained previously. Note that, as mentioned before, the matrix $\mathbf{Q}_{z}$ in (38) can be $\mathbf{0}$. If we only take the first $K=n_{\beta}$ columns of the precoder $\mathbf{Q}$, i.e., if we let $\mathbf{Q}_{n_{t} N \times K}=\mathbf{Q}_{u}$ and the signal $\overline{\mathbf{x}}_{k}$ in (13) be of size $K \times 1$ only consisting of $n_{\beta}$ independent information symbols, then the precoder encoding is equivalent to

$$
\mathbf{Q}_{n_{t} N \times K} \overline{\mathbf{x}}_{k} .
$$

Notice that, for the transmissions in (36) and (37), the corresponding precoding matrix $\mathbf{Q}_{n_{t} N \times K}=\mathbf{Q}_{u}$ is in fact a tall unitary matrix.

Remark: Let us compare the above proposed precoding with the conventional zero-padded system. For convenience, we only consider CP free MIMO-OFDM systems, i.e., $v=0$ in the above study. In the zero-padded system, among every block of $N$ symbols, $L$ of them are zeros, i.e., in one block of $N$ symbols, only $\min \left\{n_{r} N, n_{t}(N-L)\right\}$ independent information symbols are transmitted for all $n_{t}$ transmit antennas, where the reason to take the minimum is because there are only $n_{r} N$ received signals, i.e., only $n_{r} N$ linear equations and the number of independent information symbols can be solved linearly can not be above $n_{r} N$ as we explained before. In comparison, in our channel independent precoded MIMOOFDM system,

$$
\min \left\{n_{r} N, n_{t}(N-L)+\left\lfloor\frac{n_{r} N-n_{t}(N-L)}{2}\right\rfloor\right\}
$$

independent information symbols can be transmitted and linearly solved freely in one OFDM block across all $n_{t}$ transmit antennas. Clearly, one can see that, when $n_{t} \geq$ $n_{r}>n_{t}(N-L) / N$, more independent information symbols than the conventional zero-padding MIMO system can be transmitted and solved linearly with free of IBI interference in our channel independent precoded MIMO-OFDM system. A similar argument applies to an insufficient CP added MIMOOFDM system.

\section{IBI Cancellation When $n_{r}>n_{t}$}

In this case, we show that no precoding, or CP or zeropadding is needed to eliminate the IBI in the following theorem.

Theorem 2: For the insufficient CP MIMO-OFDM system with $n_{r}>n_{t}$, the total number of independent information symbols can be solved by the zero-forcing operator is $n_{t} N$, where no zero-padding or precoding is needed, when

$$
N \geq \frac{n_{t}}{n_{r}-n_{t}}(L-v) .
$$

Proof: Since all the channel coefficients are drawn from an i.i.d. continuous distribution, from Appendix I, the dimensions of channel matrix $\mathbf{C}$ and IBI matrix $\mathbf{B}$ are (almost surely)

$$
\begin{aligned}
\operatorname{dim}(\mathbf{C}) & =n_{t} N \\
\operatorname{dim}(\mathbf{B}) & =n_{t}(L-v),
\end{aligned}
$$

respectively, while the dimension of the receive signal space is $n_{r} N$. When the dimension of the receive signal space is not smaller than the sum of the dimensions of the channel matrix $\mathbf{C}$ and IBI matrix $\mathbf{B}$, i.e.,

$$
n_{t} N+n_{t}(L-v) \leq n_{r} N
$$

which is (40), it is almost surely that the signal space $\operatorname{span}\{\mathbf{C}\}$ is disjoint from the IBI space $\operatorname{span}\{\mathbf{B}\}$. Thus, all the independent information symbols can be solved by the zero-forcing operator.

\section{E. Relationships With the Existing Block Based Transmission Systems}

We now discuss the relationships of our above IA based channel independent precoding scheme with the existing block based transmission systems, namely zero-padded (ZP) only, $\mathrm{CP}-\mathrm{OFDM}$ and single carrier frequency domain equalizer (SCFDE). Without loss of generality, for convenience we only consider the SISO configuration, i.e., $n_{t}=n_{r}=1$, for the discussions below.

Let us first consider the ZP-only transmission system, see, for example [18], [19], [22], with information symbol block length $M$ and ZP length $L$ (in order to eliminate the IBI completely). Note that the ZP idea appears in [15], [16] where a precoder in the time domain is

$$
\left[\begin{array}{c}
\mathbf{I}_{K} \\
\mathbf{0}_{(N-K) \times K}
\end{array}\right]
$$

which becomes the ZP-only scheme when $K=M$ and $N-K=L$. Comparing to the IA based precoding scheme proposed in the preceding sections to completely eliminate the 
IBI, we choose $N=M+L, K=M$ in (39) with $n_{t}=1$, and $v=0$, i.e., no $\mathrm{CP}$ is used in this case. The precoding matrix $\mathbf{P}$ is designed as the $N \times K$ submatrix of the $N \times N$ DFT matrix, i.e., the $(m, n) t h$ entry of this precoding is expressed as $[\mathbf{P}]_{m, n}=(1 / \sqrt{N}) \exp (-j 2 \pi m n / N)$. As a result, we have $\mathbf{Q}_{z p}=\mathbf{W}_{N} \mathbf{P}=\left[\mathbf{I}_{K}, \mathbf{0}_{K \times L}\right]^{T}$ that is the same as the precoder (43) proposed in [15], [16].

We next consider the CP-OFDM system, i.e., the conventional OFDM system, with information symbol block length $M$, i.e., the IDFT size $M$, and CP length $L$ (in order to eliminate the IBI completely). Comparing to the IA based precoding scheme proposed in the preceding sections to completely eliminate the IBI, we choose $N=K=M$ and $v=L$, i.e., full $\mathrm{CP}$ length is used in this case. Then, the precoding matrix is the identity matrix, i.e., $\mathbf{P}=\mathbf{I}_{N}$. In other words, CPOFDM can be considered as a special case of the IA based precoding in this paper.

We finally consider the SC-FDE system, see for example [13], [14], [20], [21], with information symbol block length $M$ and CP length $L$ (in order to eliminate the IBI completely). Comparing to the IA based precoding scheme proposed in the preceding sections to completely eliminate the IBI, we choose $N=K=M$ and $v=L$, i.e., full $\mathrm{CP}$ length is used in this case. The precoding matrix $\mathbf{P}$ in this case is, however, chosen as the $N \times N$ DFT matrix, i.e., $\mathbf{P}=\mathbf{W}_{N}^{H}$. Clearly, in this case, the precoding cancels the IDFT operation, i.e., the transmission matrix $\mathbf{Q}$ is the identity matrix, and thus leads to transmit the information symbols directly. Note that when the CP length $v=L$, i.e., full (or sufficient) $\mathrm{CP}$ is used, our proposed precoded system with the precoder example (32)(33) always becomes the SC-FDE.

From the above discussions, one can see that the three existing block based transmission systems, ZP-only, CP-OFDM, and SC-FDE systems, can be all considered as special cases of our proposed IA based channel independent precoding scheme in this paper. Interestingly, as we have shown in the preceding sections, with the IA concept we may choose better channel independent precoding matrices $\mathbf{P}$ (or $\mathbf{Q}$ ) such that less number of $\mathrm{CP}$ or ZP are needed to completely eliminate the IBI caused from the ISI of the channel, when the number of information symbols is fixed. Some concrete examples are given in the simulations in the next section.

\section{Simulations}

In this section, we present some simulation results to validate our proposed IA based channel independent precoding where the precoder in (32)-(33) is used. In the meantime, some particular and concrete examples of the proposed precoded transmissions are illustrated as well. In the following figures, the SNR at the receiver is accounted.

In Fig. 1, we consider the SISO case and depict the BER performances of our proposed IA based precodings with different $\mathrm{CP}$ lengths and the numbers of independent information symbols that satisfy Theorem 1, i.e., the IBI can be completely eliminated. In Fig. 1, IA stands for the IA based precoding. The simulations in Fig. 1 also include the cases of CP-OFDM, ZP-only, and SC-FDE systems. The order of the CIR is $L=16$ in this simulation. The block length for CPOFDM, ZP-only, SC-FDE is $N=64$, i.e., 64 independent

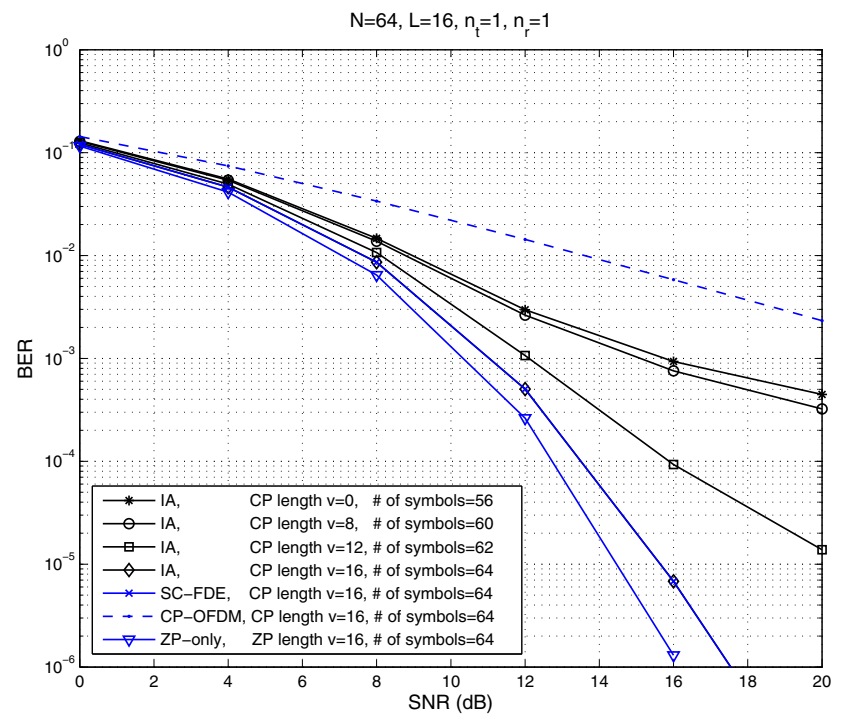

Fig. 1. BER performances of the IA based precoding, SC-FDE, CP-OFDM, ZP-only.

information symbols are sent, where full $\mathrm{CP}$ or ZP length, 16, is used. The block length for the proposed IA based precoding is $N=64$ and the CP lengths are $v=16,12,8,0$, and the corresponding numbers of independent information symbols $x_{k}(n)$ are $64,62,60,56$, respectively, according to Theorem 1. To illustrate the structure of transmission block in our IA based precoding, the transmission block for the $v=12$ case in Fig. 1 is:

$$
[\underbrace{x_{k}(52), \cdots, x_{k}(61), 0,0}_{\text {CP of length } v=12}, \underbrace{x_{k}(0), x_{k}(1), \cdots, x_{k}(61), 0,0}_{\text {data block of length } N=64}]
$$

and the transmission block for the $v=0$ case in Fig. 1 is:

$$
[\underbrace{[x_{k}(0), x_{k}(1), \cdots, x_{k}(55), \overbrace{0, \cdots, 0}^{8 \text { zeros }}}_{\text {data block of length } N=64}] .
$$

As mentioned previously in Section III-E, our IA based precoded system, when the full $\mathrm{CP}$, i.e., $v=16$, is added, coincides with SC-FDE as also shown in Fig. 1 with solid curves marked by $\diamond$ and $\times$, respectively.

For CP-OFDM, ZP-only, SC-FDE, in order to completely eliminate the IBI, the CP/ZP lengths should be at least all 16 $14,12,8$, when $64,62,60,56$ independent information symbols are sent in a data block of length $N=64$, respectively, which are clearly higher than the CP lengths $16,12,8,0$, in our proposed IA based precoding, respectively, for the last three cases. After saying so, although in all these cases, the IBI can be completely eliminated, their performances are different. One can see that the ZP-only scheme performs the best, since it achieves the full multipath diversity with the MMSE or ZF receiver [19]. In fact, this case is equivalent to the MISO case with quasi-static flat fading channels when the delay diversity code (or Toeplitz code) is used. It is theoretically shown in [25], [26] that this code achieves the full spatial diversity (corresponding to the full multipath diversity in the ISI channel case) with the ZF/MMSE receiver. 


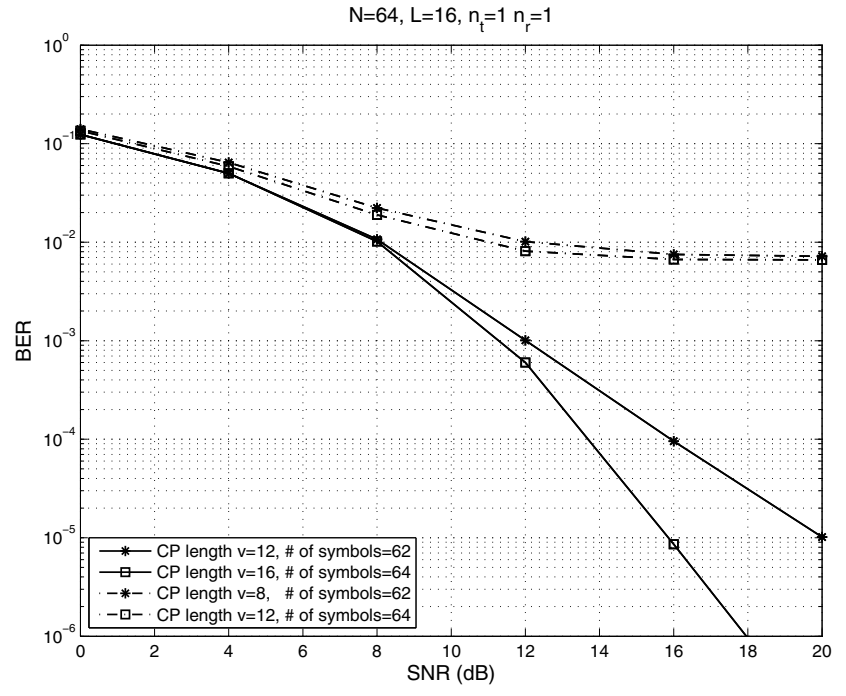

Fig. 2. SISO case: BER performances of IA based precoding of different $\mathrm{CP}$ lengths and different numbers of information symbols.

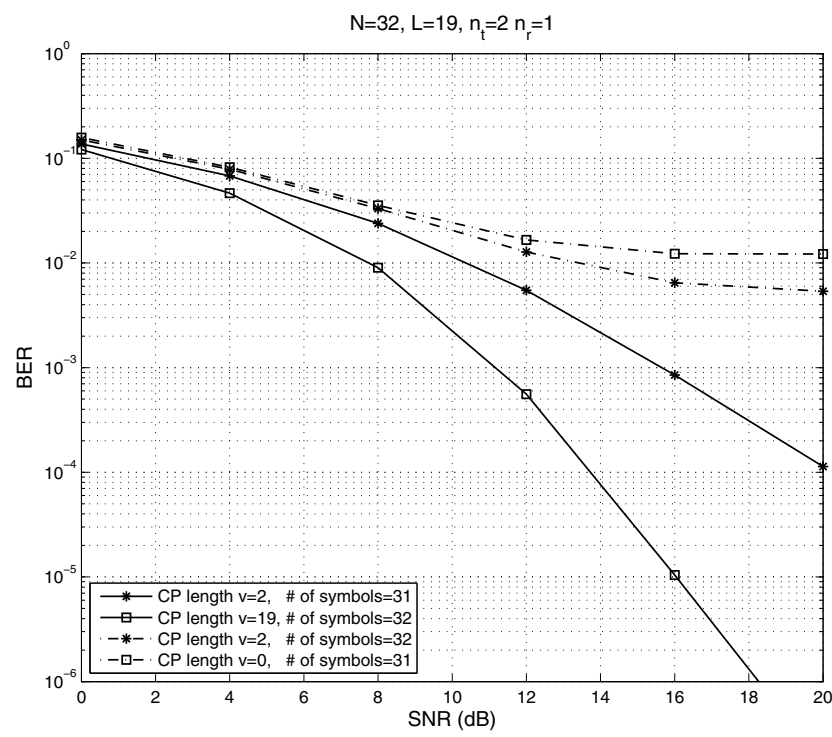

Fig. 3. MISO case: BER performances of IA based precoding of different $\mathrm{CP}$ lengths and different numbers of information symbols.

In Fig. 2, we consider the same SISO case as in Fig. 1 with two cases that do not satisfy the condition obtained in Theorem 1. These two cases are when $v=8$ and $v=12$ and the numbers of transmitted independent information symbols in one block are 62 and 64 , respectively. The BER performances for these two cases are dashed curves in Fig. 2, where one can see that error floors occur when SNR becomes high because the IBI can not be completely eliminated. Notice that when insufficient $\mathrm{CP} / \mathrm{ZP}$ is added for CP-OFDM, ZP-only or SCFDE, error floor will also occur because there is residual IBI.

In Fig. 3, we simulate the IA based precoding scheme proposed in this paper for the MISO configuration of $n_{t}=2$ and $n_{r}=1$. We consider both cases of satisfying (solid curves in Fig. 3) and not-satisfying (dashed curves in Fig. 3)

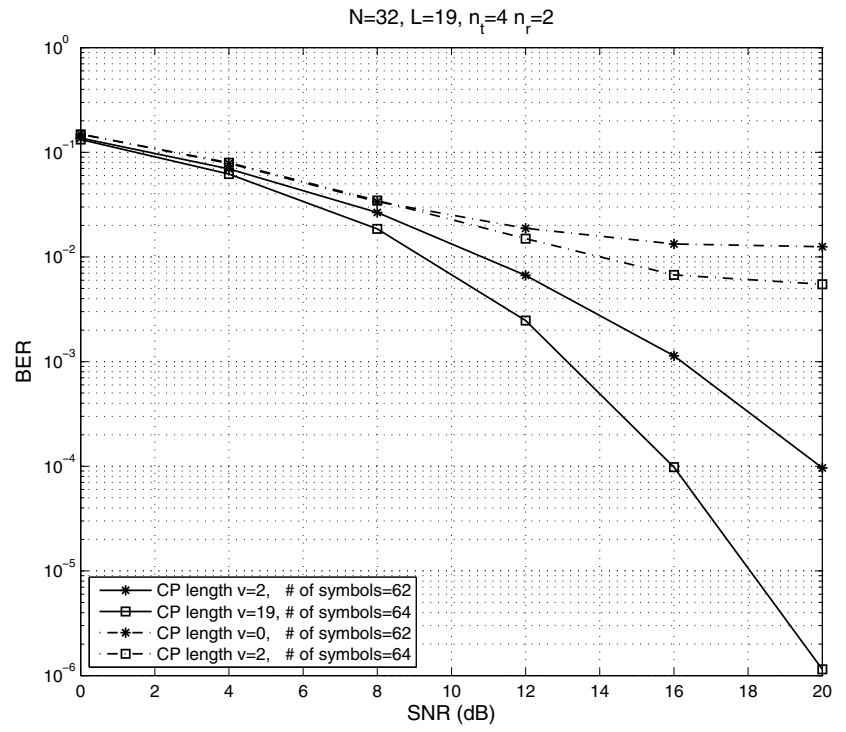

Fig. 4. MIMO case: BER performances of IA based precoding of different $\mathrm{CP}$ lengths and different numbers of information symbols.

Theorem 1. For the convenience of having the same number of transmitted independent information symbols $x_{k}(n)$ in both cases, we choose the block size $N=32$ and the CIR order $L=19$. In each case, two different CP lengths are considered. We take the $v=2$ case as an example to specify the transmission block:

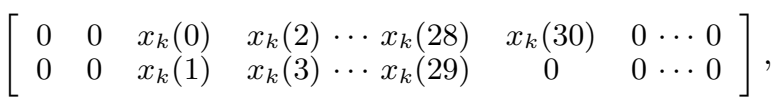

where there are 16 consecutive zeros at the end of each row, each row represents the transmission block at one transmit antenna, and the first two zeros are the $\mathrm{CP}$ of length $v=2$ in each row.

In Fig. 4, we simulate the IA based precoding scheme proposed in this paper for a MIMO configuration of four transmit and two receive antennas, i.e., $n_{t}=4$ and $n_{r}=2$. We also consider both cases of satisfying (solid curves in Fig. 4) and not-satisfying (dashed curves in Fig. 4) Theorem 1. For the convenience of having the same number of transmitted independent information symbols $x_{k}(n)$ in both cases, we also choose the block size $N=32$ and the CIR order $L=19$. In each case, two different CP lengths are considered. Again, the $v=2$ case is used as an example to specify the transmission block:

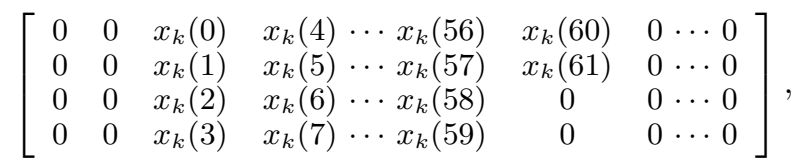

where there are 16 consecutive zeros at the end of each row for the first two rows and 17 consecutive zeros at the end of each row for the last two rows, and the first two columns of zeros are the CP of length $v=2$. Each row of (47) is for one transmit antenna. Comparing the results in Fig. 3 and Fig. 4 for the cases of two transmit and one receive antennas and four transmit and two receive antennas, respectively, we can see that the channels are the same (i.e., the CIR orders 


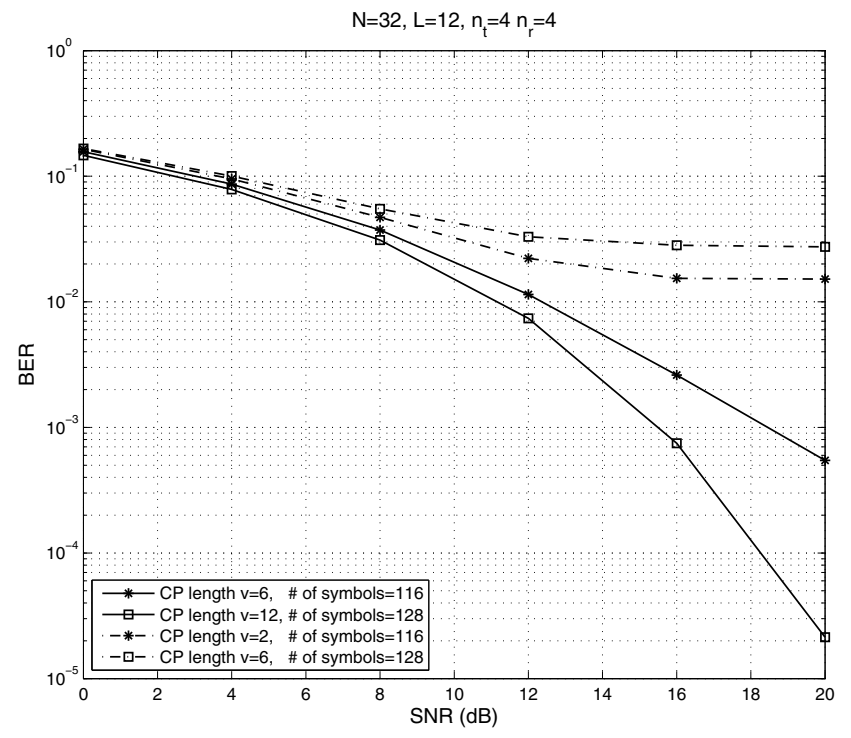

Fig. 5. MIMO case: BER performances of IA based precoding of different $\mathrm{CP}$ lengths and different numbers of information symbols.

$L$, the CP lengths $v$, the data block sizes $N$, and the ratios between the numbers of transmit and receive antennas are the same) but the number of independent information symbols can be solved for the later case almost doubles that of the former case. Although the bandwidth efficiency gets better for more antennas for our precodings, as one can see from these two figures the BER performance degrades, which may be improved by, for example, employing forward error correction coding. Another remark is that the total ZP length for each of the last two transmit antennas in (47) is already 19 that is the same as the CIR order $L$ here. In other words, for the last two transmit antennas, the IBIs do not appear. However, since there are four transmit antennas but only two receive antennas, the signals may not be solved freely as in the SISO case.

In Fig. 5, we simulate the IA based precoding scheme proposed in this paper for another MIMO configuration of $n_{t}=4, n_{r}=4$. Both cases of satisfying (solid curves in Fig. 5) and not-satisfying (dashed curves in Fig. 5) are studied. Two different $\mathrm{CP}$ lengths are considered for each case. We choose the block size $N=32$ and CIR order $L=12$. In the $v=6$ case, transmission block is specified as (48) at the bottom of the page, where the first 6 columns are the CP of length $v=6$. One can see that even more independent information symbols $x_{k}(n)$ can be sent in this configuration.

\section{CONClusion}

In this paper, we proposed a channel independent precoding for MIMO-OFDM systems with insufficient $\mathrm{CP}$ by using the notion of interference nulling that has been also actively used in interference alignment lately. We showed that our proposed precoding is more bandwidth efficient than the conventional zero-padded or CP added MIMO systems, such as, ZP-only, $\mathrm{CP}-\mathrm{OFDM}$ and SC-FDE systems, when the number of receive antennas is not more than the number of transmit antennas. When the number of receive antennas is more than the number of transmit antennas, it was shown that the IBI in an MIMOOFDM system can be completely eliminated without any $\mathrm{CP}$ or zero-padding or precoding, when the OFDM block size is not too small. The key reason behind these is that instead of making the IBI disappears completely in the conventional sufficient CP or ZP based block transmission systems, the IA based channel independent precoding proposed in this paper aligns the IBI interference subspace disjoint from the signal subspace and then the zero-forcing operator is applied to eliminate the IBI while maintain the signal. Although we only considered $\mathrm{CP}$ based block transmission systems in this paper, the theory developed in this paper can be easily generalized to ZP based block transmission systems.

\section{APPENDIX I \\ Proof of Almost Surely Full Rank Channel MATRIX C}

Let us first consider the SISO-OFDM case for channel matrix $\mathbf{C}$ in (5).

By observing the determinant expansion of the channel matrix $\mathbf{C}$ in (5), we notice that there is one term, $(h(0))^{N}$, in the determinant, which is resulted from multiplying all the $N$ entries on the main diagonal of matrix C. Furthermore, we check all other non-zero terms in the determinant expansion and conclude that all these terms are products of $N$ entries with $h(0)$ appearing strictly less than $N$ times. As a result, the expansion of the determinant of channel matrix $\mathbf{C}$ can be expressed as

$$
\begin{gathered}
\operatorname{det}(\mathbf{C})= \\
(h(0))^{N}+\tau_{1}(h(0))^{N-1}+\cdots+\tau_{N-1} h(0)+\tau_{N},
\end{gathered}
$$

where $\tau_{i}, i=1, \cdots, N$, is a polynomial of degree $i$ consisting of $h(1), \cdots, h(L)$. Since $(h(0))^{N}$ is the nonzero term in the determinant expansion in (49), $\operatorname{det}(\mathbf{C})$ is $h(0)$ 's polynomial of degree $N$. In our system model, channel coefficients $h(0), \cdots, h(L)$ are assumed i.i.d. complex Gaussian distributed, therefore the resultant polynomial in (49) is a continuously distributed random variable and furthermore $\operatorname{det}(\mathbf{C})=0$ occurs with zero probability. This means that channel matrix $\mathbf{C}$ is almost surely full rank.

$$
\left[\begin{array}{cccccccccccc}
x_{k}(104) & x_{k}(108) & x_{k}(112) & 0 & 0 & 0 & x_{k}(0) & \cdots & x_{k}(112) & 0 & 0 & 0 \\
x_{k}(105) & x_{k}(109) & x_{k}(113) & 0 & 0 & 0 & x_{k}(1) & \cdots & x_{k}(113) & 0 & 0 & 0 \\
x_{k}(106) & x_{k}(110) & x_{k}(114) & 0 & 0 & 0 & x_{k}(2) & \cdots & x_{k}(114) & 0 & 0 & 0 \\
x_{k}(107) & x_{k}(111) & x_{k}(115) & 0 & 0 & 0 & x_{k}(3) & \cdots & x_{k}(115) & 0 & 0 & 0
\end{array}\right]
$$


For the general MIMO-OFDM case with channel matrix $\mathbf{C}$ in (11), every channel coefficient $h(i)$ above is replaced by a channel coefficient matrix $\mathbf{H}(i)$ in (9) of size $n_{r} \times n_{t}$ with i.i.d. components $h_{m n}(i)$. In this case, we permute the block matrix $\mathbf{C}$ row and column wisely such that the permuted matrix is another block matrix where the $(m, n)$ th subblock is the SISO-OFDM matrix $\mathbf{C}_{m n}$ in (5) with channel coefficients $h_{m n}(l)$. As what is just proved, every such a subblock $\mathbf{C}_{m n}$ has full rank almost surely, i.e., its columns or rows are almost surely linearly independent. Since all these subblocks $\mathbf{C}_{m n}$ are independent each other and all complex Gaussian distributed, the overall block matrix C's rows or columns are almost surely linearly independent, i.e., full rank.

\section{APPENDIX II \\ Proof of Almost Surely $\operatorname{span}\{\mathbf{C Q}\} \cap \operatorname{span}\{\mathbf{B Q}\}=\{\mathbf{0}\}$}

We also first consider the SISO-OFDM case, the IBI matrix $\mathbf{B}$ is specified in (3) and (4) and the channel matrix $\mathbf{C}$ is defined in (5). We restate that $n_{1}=\lfloor(L-v) / 2\rfloor, n_{\alpha}=N-$ $L+v$ and $n_{\beta}=n_{1}+n_{\alpha}$. Consider the design example with $\mathbf{Q}_{z}=\mathbf{0}$, i.e., $\mathbf{Q}^{\prime}=\left[\mathbf{e}_{n_{\alpha}+1}, \cdots, \mathbf{e}_{n_{\beta}}, \mathbf{e}_{n_{1}}, \cdots, \mathbf{e}_{n_{\alpha}}, \mathbf{0}, \cdots, \mathbf{0}\right]$, proposed in this paper previously.

We next first show that $\operatorname{span}\left\{\mathbf{C Q}^{\prime}\right\} \bigcap \operatorname{span}\left\{\mathbf{B Q}^{\prime}\right\}=\{\mathbf{0}\}$ holds almost surely for this particular precoder $\mathbf{Q}^{\prime}$ and then we show that it is also true for a general precoder $\mathbf{Q}$.

Let $\mathbf{b}_{i}$ and $\mathbf{c}_{i}$ denote the $i$ th columns of matrices $\mathbf{B}$ and $\mathbf{C}$, respectively. Without loss of generality, assume that $\left\{\mathbf{c}_{1}, \cdots, \mathbf{c}_{n_{\beta}}\right\}$ is a maximal set of $n_{\beta}$ independent columns in $\mathbf{C Q}^{\prime}$, and $\left\{\mathbf{b}_{n_{\alpha}+1}, \cdots, \mathbf{b}_{n_{\beta}}\right\}$ is a maximal set of $n_{1}$ independent columns in $\mathbf{B Q}^{\prime}$. Then, let $\mathbf{B}^{\prime}=\left[\mathbf{b}_{n_{\alpha}+1}, \cdots, \mathbf{b}_{n_{\beta}}\right]$ that is an $N \times n_{1}$ matrix and $\mathbf{C}^{\prime}=\left[\mathbf{c}_{1}, \cdots, \mathbf{c}_{n_{\beta}}\right]$ that is an $N \times n_{\beta}$ matrix. We construct an $N \times\left(n_{\beta}+n_{1}\right)$ matrix $\mathbf{H}_{Q}=\left[\begin{array}{ll}\mathbf{B}^{\prime} & \mathbf{C}^{\prime}\end{array}\right]$. Notice that when $L-v$ is even, $n_{\beta}+n_{1}=N, \mathbf{H}_{Q}$ is a square matrix. When $L-v$ is odd, $n_{\beta}+n_{1}=N-1$. But for this proof we only need to show that $\mathbf{H}_{Q}$ is full column rank, i.e., the column rank of $\mathbf{H}_{Q}$ is $n_{1}+n_{\beta}$, almost surely.

The explicit structures of $\mathbf{H}_{Q}$ are separately introduced in the following two equations, since the change of $n_{1}$ results in a change in the form of $\mathbf{H}_{Q}$.

(i) When $n_{1} \geq v, \mathbf{H}_{Q}$ is expressed in (50), and (ii) When $n_{1}<v, \mathbf{H}_{Q}$ is given in (51).

We only prove that $\mathbf{H}_{Q}$ has a full column rank when $n_{1} \geq v$ here. Applying the same argument, the case when $n_{1}<v$ can be proved too.

First, we denote the upper-left $n_{1} \times n_{1}$ submatrix in (50) as

$$
\mathbf{H}_{1}=\left[\begin{array}{ccc}
h(L) & \ldots & h\left(L-n_{1}+1\right) \\
& \ddots & \vdots \\
& & h(L)
\end{array}\right] .
$$

Since $\operatorname{det}\left(\mathbf{H}_{1}\right)=(h(L))^{n_{1}}$, this matrix is almost surely full rank.

Next, we perform column elementary operations on $\mathbf{H}_{Q}$ in (50). Using the first $n_{1}$ columns, all the non-zero entries from the $\left(n_{1}+1\right)$ th to the last column of the first $n_{1}$ rows can

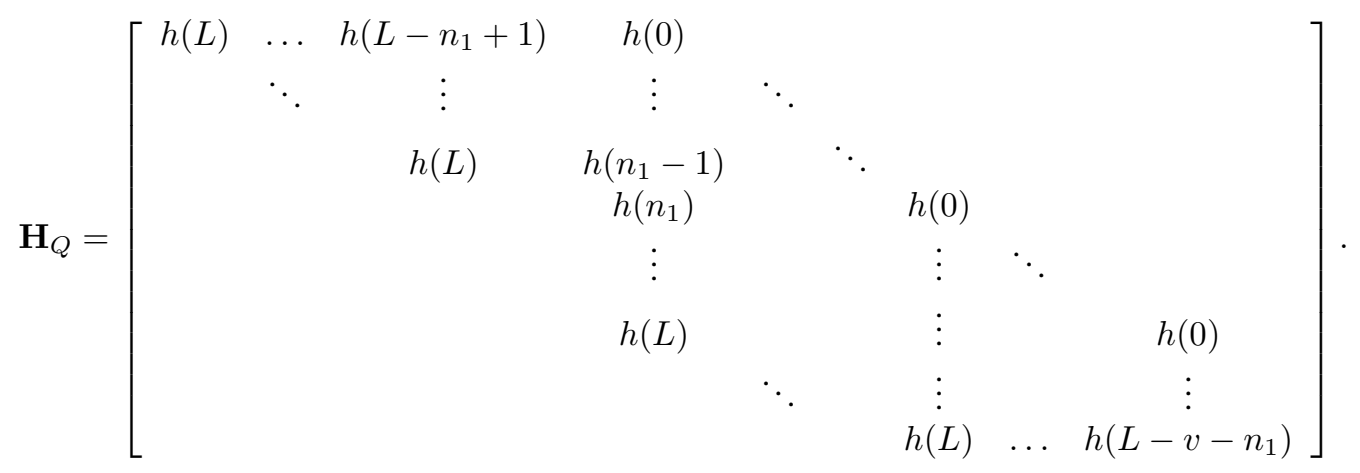

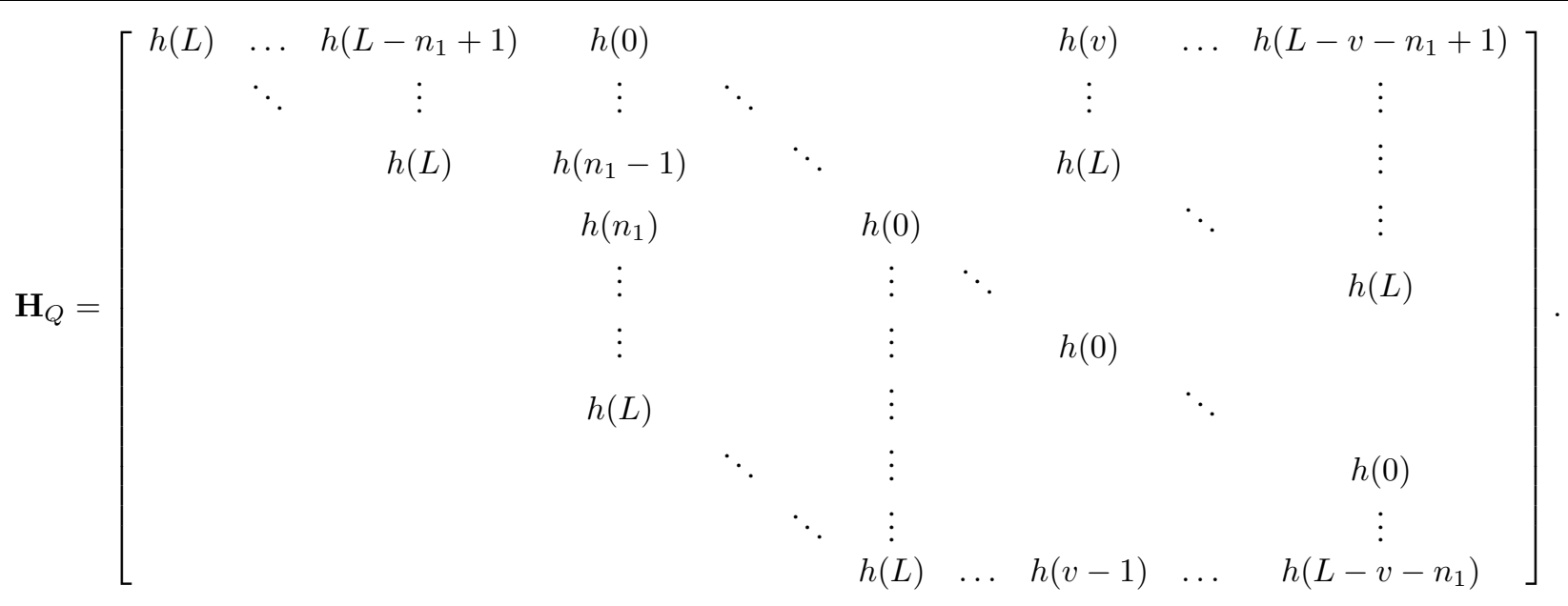


be eliminated without influencing the entries below the $n_{1}$ th row. Thus, we transform $\mathbf{H}_{Q}$ into $\mathbf{H}_{Q}^{\prime}$ which has the identical column rank shown in (53) at the bottom of the page.

Take the entries in $\mathbf{H}_{Q}^{\prime}$ with both row and column indices belonging to $\left\{n_{1}+1, \cdots, n_{1}+n_{\beta}\right\}$ to form the Toeplitz submatrix $\mathbf{H}_{2}$ :

$$
\mathbf{H}_{2}=\left[\begin{array}{ccccc}
h\left(n_{1}\right) & \ldots & h(0) & & \\
\vdots & \ddots & & \ddots & \\
h(L) & & \ddots & & h(0) \\
& \ddots & & \ddots & \vdots \\
& & h(L) & \ldots & h\left(n_{1}\right)
\end{array}\right] .
$$

Define

$$
\mathbf{H}_{a}=\left[\begin{array}{cc}
\mathbf{H}_{1} & \mathbf{0} \\
\mathbf{0} & \mathbf{H}_{2}
\end{array}\right] .
$$

$\mathbf{H}_{a}$ is just $\mathbf{H}_{Q}^{\prime}$ when $L-v$ is even or $\mathbf{H}_{a}$ is the $\left(n_{1}+n_{\beta}\right) \times$ $\left(n_{1}+n_{\beta}\right)$ submatrix in $\mathbf{H}_{Q}^{\prime}$ by deleting the last row of $\mathbf{H}_{Q}^{\prime}$ when $L-v$ is odd.

Now compute the determinant of $\mathbf{H}_{2}$. We similarly expand the determinant as a polynomial of $h\left(n_{1}\right)$,

$$
\begin{gathered}
\operatorname{det}\left(\mathbf{H}_{2}\right)= \\
\left(h\left(n_{1}\right)\right)^{n_{\beta}}+\gamma_{1}\left(h\left(n_{1}\right)\right)^{n_{\beta}-1}+\cdots+\gamma_{N-1} h\left(n_{1}\right)+\gamma_{N}
\end{gathered}
$$

where $\gamma_{i}, i=1, \cdots, n_{\beta}$ is a polynomial of degree $i$ consisting of $h(0), \cdots, h\left(n_{1}-1\right), h\left(n_{1}+1\right), \cdots, h(L)$. Thus, $\operatorname{det}\left(\mathbf{H}_{2}\right)=0$ with zero probability, i.e., $\mathbf{H}_{2}$ is almost surely full rank and hence $\mathbf{H}_{a}$ is almost surely full rank or $\mathbf{H}_{Q}^{\prime}$ and $\mathbf{H}_{Q}$ are almost surely full column rank. Therefore, we have $\operatorname{span}\left\{\mathbf{C Q}^{\prime}\right\} \bigcap \operatorname{span}\left\{\mathbf{B Q}^{\prime}\right\}=\{\mathbf{0}\}$ almost surely.

For a general precoder $\mathbf{Q}$ that satisfies all the requirements in Theorem 1, we can give a structure of such a precoder:

$$
\mathbf{Q}=\left[\begin{array}{cc}
\mathbf{Q}_{u} & \mathbf{0} \\
\mathbf{0} & \mathbf{0}
\end{array}\right] \text {. }
$$

where $\mathbf{Q}_{u}$ is an $n_{\beta} \times n_{\beta}$ nonzero submatrix. Due to the special structure of $\mathbf{Q}^{\prime}$, it can be easily seen that each column of $\mathbf{C Q}$ is a linear combination of the maximal independent column set $\left\{\mathbf{c}_{1}, \cdots, \mathbf{c}_{n_{\beta}}\right\}$ of $\mathbf{C Q}^{\prime}$ and each column of $\mathbf{B Q}$ is a linear combination of $\left\{\mathbf{b}_{n_{\alpha}+1}, \cdots, \mathbf{b}_{n_{\beta}}\right\}$. This means $\operatorname{span}\{\mathbf{C Q}\} \bigcap \operatorname{span}\{\mathbf{B Q}\}=\{\mathbf{0}\}$ also holds almost surely.

The general MIMO-OFDM case can be proved similarly by using the same argument as in Appendix I.

\section{REFERENCES}

[1] J.-B. Lim, C.-H. Choi, and G.-H. Im, "MIMO-OFDM with insufficient cyclic prefix," IEEE Commun. Lett., vol. 10, pp. 356-358, May 2006.

[2] M. Grossmann, C. Schneider, and R. S. Thoma, "Turbo equalisation for MIMO-OFDM transmission with insufficient guard interval," in Proc. 2006 International Zurich Seminar Commun., pp. 114-117.

[3] J. Guo, S. Ren, and H. Xiang, "A non-cyclic prefixed MIMO-OFDM system based on a recursive algorithm of joint channel estimation and data detection," in Proc. 2008 International Conf. Wireless Commun., Netw. Mobile Comput., pp. 1-6.

[4] X. Sun, L. J. Cimini, L. J. Greenstein, and D. S. Chan, "ICI/ISI aware beamforming for MIMO-OFDM wireless system," in Proc. 2009 Conf. Inf. Sciences Syst., pp. 103-107.

[5] V. Kotzsch, W. Rave, and G. Fettweis, "ISI analysis in network MIMO OFDM systems with insufficient cyclic prefix length," in Proc. 2010 International Symp. Wireless Commun. Syst., pp. 189-193.

[6] S. Chern, J. Chen, and C. Wu, "Novel frequency-domain DFE equalizer with oblique projection for CP-free space-time block coded MIMOOFDM systems," in Proc. 2009 International Symp. Intelligent Signal Process. Commun. Syst., pp. 541-545.

[7] K.-W. Cheong and J. M. Cioffi, "Precoder for DMT with insufficient cyclic prefix," in Proc. 1998 IEEE Int. Conf. Commun., pp. 339-343.

[8] S. Trautmann and N. J. Fliege, "Perfect equalization for DMT systems without guard interval," IEEE J. Sel. Areas Commun., vol. 20, pp. $987-$ 996, June 2002.

[9] C.-J. Park and G.-H. Im, "Efficient DMT/OFDM transmission with insufficient cyclic prefix," IEEE Commun. Lett., vol. 8, pp. 576-578, Sep. 2004.

[10] S. A. Jafar and S. Shamai, "Degree of freedom region for the MIMO X channel," IEEE Trans. Inf. Theory, vol. 54, pp. 151-170, Jan. 2008.

[11] V. R. Cadambe and S. A. Jafar, "Interference alignment and the degrees of freedom for the $K$ user interference channel," IEEE Trans. Inf. Theory, vol. 54, pp. 3425-3441, Aug. 2008.

[12] T. Gou and S. A. Jafar, "Degree of freedom of the $K$ user $M \times N$ MIMO interference channel," IEEE Trans. Inf. Theory, vol. 56, pp. 6040-6057, Dec. 2010.

[13] H. Sari, G. Karam, and I. Jeanclaude, "Transmission techniques for digital terrestrial TV broadcasting," IEEE Commun. Mag., pp. 100-109, Feb. 1995.

[14] M. V. Clark, "Adaptive frequency-domain equalization and diversity combining for broadband wireless communications," IEEE J. Sel. Areas Commun., vol. 16, pp. 1385-1395, Oct. 1998.

[15] X.-G. Xia, "Applications of nonmaximally decimated multirate filterbanks in partial response channel ISI cancellation," in Proc. 1996 IEEE Third Time-Frequency Time-Scale Symp.

[16] X.-G. Xia, "New precoding for intersymbol interference cancellation using nonmaximally decimated multirate filterbanks with ideal FIR equalizers," IEEE Trans. Signal Process., vol. 45, pp. 2431-2441, Oct. 1997.

[17] X.-G. Xia, "Precoded and vector OFDM robust to channel spectral nulls and with reduced cyclic prefix length in single transmit antenna systems," IEEE Trans. Commun., vol. 49, pp. 1363-1374, Aug. 2001.

[18] Z. Wang and G. B. Giannakis, "Wireless multicarrier communications: where Fourier meets Shannon," IEEE Signal Process. Mag., vol. 47, pp. 29-48, May 2000.

[19] C. Tepedelenlioglu, "Low complexity linear equalizers with maximum multipath diversity for zero-padded transmissions," in Proc. 2003 Int. Conf. Acoustics, Speech, Signal Process., vol. IV, pp. 636-639.

$$
\mathbf{H}_{Q}^{\prime}=\left[\begin{array}{ccccccccc}
h(L) & \ldots & h\left(L-n_{1}+1\right) & 0 & & \ldots & \ldots & \ldots & 0 \\
& \ddots & \vdots & \vdots & & & & & \vdots \\
& & h(L) & 0 & & \ldots & \ldots & \ldots & 0 \\
& & & h\left(n_{1}\right) & \ldots & & h(0) & & \\
& & & \vdots & & & \vdots & \ddots & \\
& & & h(L) & & \vdots & & h(0) \\
& & & & \ddots & & \vdots & & \vdots \\
& & & & & h(L) & \ldots & h\left(L-v-n_{1}\right)
\end{array}\right]
$$


[20] D. Falconer, S. L. Ariyavisitakul, A. Benyamin-Seeyar, and B. Eidson, "Frequency domain equalization for single-carrier broadband wireless systems," IEEE Commun. Mag., vol. 40, no. 4, pp. 58-66, Apr. 2002.

[21] F. Pancaldi, G. M. Vitetta, R. Kalbasi, N. Al-Dhahir, M. Uysal, and H. Mheidat, "Single-carrier frequency domain equalization," IEEE Signal Process. Mag., vol. 25, no. 5, pp. 37-56, Sep. 2008.

[22] S. Ohno, "Performance of single-carrier block transmissions over multipath fading channels with linear equalization," IEEE Trans. Signal Process., vol. 54, pp. 3678-3687, Oct. 2006.

[23] Y. Fu, C. Tellambura, and W. A. Krzymien, "Transmitter precoding for ICI reduction in closed-loop MIMO OFDM systems," IEEE Trans. Veh. Technol., vol. 56, no. 1, pp. 115-125, Jan. 2007.

[24] Recommendation ITU-R M. 1225, International Telecommunication Union, Guidelines for Evaluation of Radio Transmission Technologies for IMT-2000, Feb. 1997.

[25] J.-K. Zhang, J. Liu, and K. M. Wong, "Linear Toeplitz space time block codes," in Proc. 2005 IEEE Int. Symp. Inf. Theory.

[26] J. Liu, J.-K. Zhang, and K. M. Wong, "Full-diversity codes for MISO systems equipped with linear or ML detectors," IEEE Trans. Inf. Theory, vol. 54, pp. 4511-4527, Oct. 2008.

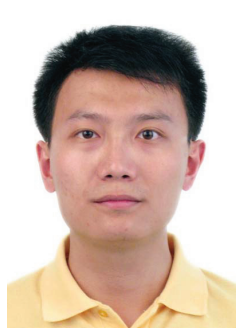

Yuansheng Jin received the B.S. degree from Southwest Jiaotong University, Chengdu, China, in 2007, and the M.S. degree from the University of Science and Technology of China, Hefei, in 2010, both in electrical engineering. He is currently pursuing the $\mathrm{Ph}$. D degree at the University of Delaware, Newark. His current research interest lies in the area of precoding for MIMO-OFDM systems.

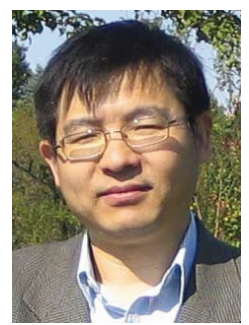

Xiang-Gen Xia (M'97,S'00,F'09) received his B.S. degree in mathematics from Nanjing Normal University, Nanjing, China, and his M.S. degree in mathematics from Nankai University, Tianjin, China, and his Ph.D. degree in Electrical Engineering from the University of Southern California, Los Angeles, in 1983, 1986, and 1992, respectively.

$\mathrm{He}$ was a Senior/Research Staff Member at Hughes Research Laboratories, Malibu, California, during 1995-1996. In September 1996, he joined the Department of Electrical and Computer Engineering, University of Delaware, Newark, Delaware, where he is the Charles Black Evans Professor. His current research interests include space-time coding, MIMO and OFDM systems, digital signal processing, and SAR and ISAR imaging. Dr. Xia has over 240 refereed journal articles published and accepted, and 7 U.S. patents awarded and is the author of the book Modulated Coding for Intersymbol Interference Channels (New York, Marcel Dekker, 2000).

Dr. Xia received the National Science Foundation (NSF) Faculty Early Career Development (CAREER) Program Award in 1997, the Office of Naval Research (ONR) Young Investigator Award in 1998, and the Outstanding Overseas Young Investigator Award from the National Nature Science Foundation of China in 2001. He also received the Outstanding Junior Faculty Award of the Engineering School of the University of Delaware in 2001. He is currently an Associate Editor of the IEEE TRANSACTIONS ON WIRELESS Communications, IEEE Transactions on Signal Processing, Signal Processing (China), and the Journal of Communications and Networks (JCN). He was a guest editor of Space-Time Coding and Its Applications in the EURASIP Journal of Applied Signal Processing in 2002. He served as an Associate Editor of the IEEE TRANSACTIONS ON SIGNAL PROCESSING during 1996 to 2003, the IEEE TRANSACTIONS ON Mobile CoMputing during 2001 to 2004, IEEE TRANSACTIONS ON VEHICULAR TECHNOLOGY during 2005 to 2008, the IEEE SignAL PROCESSING LETTERS during 2003 to 2007, Signal Processing (EURASIP) during 2008 to 2011, and the EURASIP Journal of Applied Signal Processing during 2001 to 2004. Dr. Xia served as a Member of the Signal Processing for Communications Committee from 2000 to 2005 and a Member of the Sensor Array and Multichannel (SAM) Technical Committee from 2004 to 2009 in the IEEE Signal Processing Society. He serves as IEEE Sensors Council Representative of IEEE Signal Processing Society (from 2002) and served as the Representative of IEEE Signal Processing Society to the Steering Committee for IEEE Transactions on Mobile Computing during 2005 to 2006. Dr. Xia is Technical Program Chair of the Signal Processing Symp., Globecom 2007 in Washington D.C. and the General Co-Chair of ICASSP 2005 in Philadelphia. 